\section{Successful treatment of idiopathic knuckle pads with a combination of high-dose salicylic acid and urea topical keratolytics: $\mathrm{A}$ case report}

\author{
Davide Sogliani, ${ }^{1}$ Chiara Mura, ${ }^{1}$ \\ Giorgio Tamborrini²
}

${ }^{1}$ Department of Biology and Biotechnology, University of Pavia, Italy; ${ }^{2} \mathrm{UZR}$, Ultrasound Center Basel, Switzerland

\begin{abstract}
Knuckle pads are benign subcutaneous and usually hyperkeratotic fibromas for which no specific treatments exist. Unspecific treatments are, most of the time, ineffective and a wait-and-see policy is often recommended to patients. However, especially in adolescents, knuckle pads are often poorly tolerated for cosmetic reasons, potentially causing embarrassment and social anxiety. Here we present the case of a young adult successfully treated with a combination of high-dose salicylic acid and urea topical keratolytics. In addition, we provide ideal diagnostic images obtained via high-resolution ultrasonography and histological features that can be used by medical practitioners to better distinguish knuckle pads from other proximal interphalangeal/metacarpophalangeal joint diseases as also from other diseases inducing swelling of periarticular soft tissues.
\end{abstract}

\section{Introduction}

Knuckle pads (KPs) or Garrod's nodules are benign subcutaneous fibrotic nodules located in the extensor surface of feet and/or more frequently in finger joints. ${ }^{1-3}$ Clinically, they are well-circumscribed, non-compressible, freely movable, wartlike lesions involving mostly the dorsal aspect of proximal interphalangeal (PIP) and more rarely metacarpophalangeal (MCP) joints. ${ }^{4,5}$ Usually, KPs are painless, asymptomatic (i.e. no functional effects on the joints like reduced flexibility or alterations of tendons), and characterized by a progressive and gradual growth that leads them to reach their final size (up to $40 \mathrm{~mm}$ in diameter). ${ }^{2,4,5}$ Primarily (idiopathic) KPs are the most common in children and young adults, while in older patients KPs are often associated with fibrosing disorders like Dupuytren's contracture, Peyronie's dis- ease, and Ledderhose disease (i.e., plantar fibromatosis). Secondary (acquired) KPs, instead, appear following the recurrence of frictional/traumatic events involving the joints, like those typical of some professional and athletic activities. ${ }^{2,3,5}$ The diagnosis is clinical with warts, xanthomas, rheumatoid nodules, gouty tophi, neurofibromas, Bouchard's and Heberden's nodes, synovial cysts, and retained foreign bodies reported as a differential diagnosis. ${ }^{2}$ In the case of diagnostic doubt, ultrasonography, histology (if sonography is not clear definite), and plain radiograph enhance the information gathering process reducing the risk of misdiagnosis..$^{2-4}$ On ultrasound, KPs appear as noncompressible, dome-shaped, hypoechoic nodules with absent or peripheral vascularization at Doppler analysis, while histology reveals increased (myo-)fibroblasts proliferation often associated with hyperkeratosis. ${ }^{1-4,6}$

Owing to the absence of specific and effective treatments, a stressful wait-andsee approach is usually recommended. ${ }^{1,4}$ Surgery has also been used, usually without leading to an aesthetic improvement with recurrences, post-operational loss of joint flexibility, scars, and keloids as possible side effects. ${ }^{1,6,7}$ Recently, few treatments are turning out to be effective, but they are mostly based on case reports and are often moderately to highly invasive. ${ }^{1,3,5}$ Case reports-based treatments are associated with a low level of evidence and, especially in painless and non-pathogenic conditions, priority should be given to non-invasive methods. Previous reports have shown conflicting results regarding the use of keratolytics in the treatment of KPs, ${ }^{1,3,7,8}$ usually without leading to complete resolution. On the contrary, we report here a potential resolutive treatment for idiopathic KPs based on the combination of two common keratolytics, i.e., urea and salicylic acid, administered at high dosages.

\section{Case Report}

A 15-years-old healthy boy developed nodules on PIP joints of the left-hand fingers (fifth finger excluded; Figure 1A), as also in all the fingers of both feet since autumn 2011 (Figure 2). No traumatic events were reported and the family history as also the patient's medical history did not suggest a possible genetic predisposition for KPs or fibrosing disorders.

From July 2012 to August 2013, the patient received independent KP diagnosis by dermatologists and a hand surgeon. All dermatologists prescribed several types of thera-
Correspondence: Davide Sogliani, Department of Biology and Biotechnology, University of Pavia, Via A. Ferrata 9, 27100 Pavia, Italy.

Tel.: +39.346.7419123.

E-mail: davide.sogliani@gmail.com

Key words: Knuckle pads, Garrod's nodes, fibromas, keratolytics, high-resolution ultrasonography.

Acknowledgments: We are grateful to all the MDs that, since 2012, have contributed to the formulation of KP diagnosis and for the various treatments provided to the patient. We kindly thank Prof. Claudio Luchini (University of Verona) for the histopathological investigation. We also thank Fabio Cavalletti for figure preparation.

Contributions: DS conceived and wrote the study with support from all authors. GT helped in supervising the work. All authors provided significant intellectual contribution to the work, read and approved the final manuscript.

Conflict of interest: The authors declare no potential conflict of interest.

Funding: None.

Ethics approval and consent to publication: Not applicable

Availability of data and materials: Not applicable. Info available by corresponding author.

Please cite this article as: Sogliani D, Mura $C$, Tamborrini G. Successful treatment of idiopathic knuckle pads with a combination of high-dose salicylic acid and urea topical keratolytics: A case report. Dermatol Rep 2021:13:9072.

Received for publication: 11 January 2021. Revision received: 10 February 2021.

Accepted for publication: 11 February 2021.

This work is licensed under a Creative Commons Attribution-NonCommercial 4.0 International License (CC BY-NC 4.0).

(C) Copyright: the Author(s), 2021

Licensee PAGEPress, Italy

Dermatology Reports 2021; 13:9072

doi:10.4081/dr.2021.9072

pies, while the surgeon proposed a surgical intervention, emphasizing the risk of possible recurrence as well as other side effects such as scars and loss of joint flexibility. Therapies were several and mainly based on 5\% urea cream application for 3 months, 25\% urea cream application for 40 days, different wart removal products (i.e., lactic acid, glycolic acid, and salicylic acids) for 40 days, and 
cryotherapy. All ended unsuccessfully. In mid-2014, resigned, the patient stopped treating the lesions which remained unchanged in shape and size.

\section{Ultrasonographic and histologic features of Knuckle pads}

Recently, ultrasound (hereafter, US) and histological investigations were performed on the refractory right big toe KP (Figure 2; black arrow) and, based on these new findings, the original diagnosis was confirmed. US (13 MHz operating frequency) of the plaque revealed a subcutaneous, uncompressible, and hypoechoic fibroma, while Doppler-mode US showed the absence of internal vascularization (see Supplementary Files SF_1). Sometimes, high-resolution US investigation might be necessary in order to increase the resolution of the diagnostic images. In dermatology, the optimal observation of surface structures - as KPs are - is usually reached with transducers working between 18 and $24 \mathrm{MHz} .{ }^{9}$ For this reason, we provided here a series of US images of KPs (see Supplementary Files SF_2) acquired at high resolution (15-18 MHz operating frequency), thus expanding the current knowledge on the ultrasonographic characteristics of such nodules., $, 4,10-12$ Histological examination showed dermal fibrosis and the predominance of the stratum corneum layer (Figure 3).

\section{Resolutive therapy}

At the end of 2017, the patient autonomously decided to restart a new treatment combining the active ingredients of those therapies that had worked partially and momentarily (i.e., urea and salicylic acid). He also decided to administer topically high-dose urea/salicylic acid preparations, as well as to increase the frequency of urea treatments. The administration of those keratolytics was not suspended after 40-90 days of treatment as originally recommended. Novel therapy was based on two applications per day of $25 \%$ urea-based emollient-keratolytic cream, one overnight application per day of $40 \%$ urea-based emollient-keratolytic cream, and one application per day of $30 \%$ salicylic acid ointment. The three major KPs (i.e., left-hand thumb and big toes) were treated overnight under occlusion at least five times a week. Great attention was paid to not causing trauma to the nodules both during the removal of dead skin and in daily life. Depending on the size of the KPs, 4 to 6 months were required for the total or quasi-total removal of the lesions. All the four KPs of the hand disappeared completely, with great cosmetic and psychological benefit (Figure 1B). All feet KPs disappeared or were greatly reduced both in diameter and thickness (Figure 2), with no recurrences observed after years from the end of the treatment.

The only KP partially refractory to the therapy was that of the right big toe that was anyway lowered by a few millimeters.

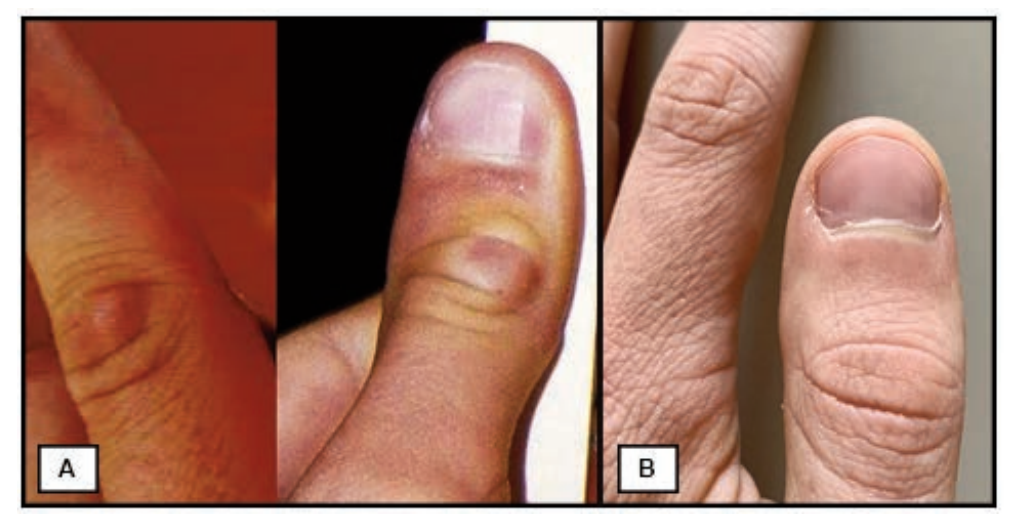

Figure 1. Knuckle Pads on proximal interphalangeal joints of the left-hand fingers before resolutive treatment, $A)$. Situation at the end of the resolutive topical therapy based on $25-40 \%$ urea and $30 \%$ salicylic acid keratolytics B).

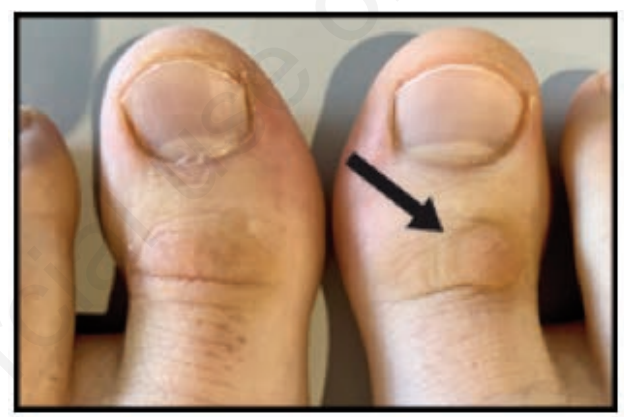

Figure 2. Knuckle Pad (black arrow) on the right big toe partially refractory to the keratolytic treatment. The Knuckle Pad that was originally present on the left big toe completely disappeared after treatment.

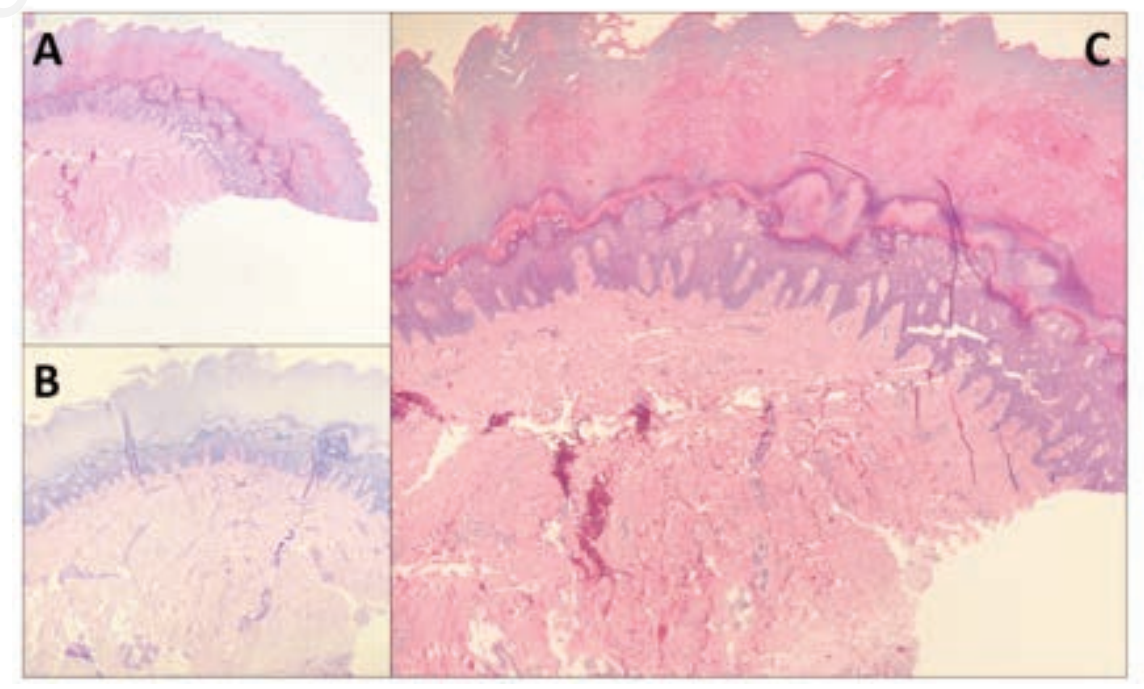

Figure 3. Knuckle Pad histological examination: A) Hematoxylin-eosin (H\&E) staining shows the histological architecture with mild dermal fibrosis and the predominance of the corneum layer; B) Periodic acid-Schiff (PAS) staining shows the dermal-epidermal interface and the dermal adnexa (original magnification: $8 x$ ); C) $H \& E$, central detail of $A$, at a higher magnification (original magnification: 20x). 


\section{Discussion and Conclusions}

Knuckle pads are recognized as refractory cutaneous lesions and usually, they do not disappear over time whether they are treated or not. Spontaneous resolution of primarily KPs has never been described, although in rare cases they can become smaller without disappearing. ${ }^{6}$ In the case of acquired KPs, they may disappear after elimination of the source of friction/trauma. ${ }^{3}$ Few case studies reporting after-treatment resolution of KPs have been reported in scientific literature and are often based on incisive therapies mostly carried out after several more or less moderate attempts to cure them. In clinical practice, there are many reports of refractory cases finally treated with high-dose and/or longlasting therapies. Besides, in KPs treatment, what works for one patient often does not work in another, suggesting a possible heterogeneous pathogenesis and indicating that many attempts should be made in trying to resolve this condition. However, in clinical practice the trail-an-error approach is nonoptimal, and the definition of gold standard therapies must remain a priority. Moreover, especially in painless non-pathogenic disorders, like Garrod's nodules, patients should start being treated with non-invasive therapies, possibly avoiding surgery, or leaving it as a last resort. Here, alongside high-resolution ultrasound and histologic images of KPs, we have presented a possible novel keratolytic-based first-line therapy which has proven to be resolutive, painless, and risk-free. Additionally, we can conclude that ultrasonography should be considered as the ideal front-line tool for diagnostic imaging of KPs, and the detailed images we have provided in this manuscript perfectly complement what is summarized in important published works on that topic., ${ }^{2,10-12}$ To conclude, this knowledge not only can make physicians familiar with the typical ultrasonographic appearance of KPs, thus potentially reducing the risk of misdiagnosis, but it can also play a role in medical education about this "forgotten skin condition".

\section{References}

1. Herold M, Russe-Wilflingseder K. Knuckle pads - A common problem but good to treat by Laser. In: AIP Conference Proceedings. 2010;1226:179-83.

2. Tamborrini G, Gengenbacher M, Bianchi S. Knuckle pads - a rare finding. J Ultrason 2012;12:493-8.

3. Hasbún C, Sandoval M, Curi M. A novel treatment for idiopathic knuckle pads with cantharidin-podophylotoxinsalicylic acid. Pediatr Dermatol 2019;36:544-5.

4. De Keersmaeker A, Vanhoenacker F. Imaging Features of Knuckle Pads. J
Belgian Soc Radiol 2016;100:1-2.

5. Weiss E, Amini S. A novel treatment for knuckle pads with intralesional Fluorouracil. Arch Dermatol 2007;143:1458-60.

6. Caroli A, Zanasi S, Marcuzzi A, et al. Epidemiological and structural findings supporting the fibromatous origin of dorsal knuckle pads. J Hand Surg Br 1991;16:258-62.

7. Paller AS, Hebert AA. Knuckle pads in children. Am J Dis Child 1986;140:9157.

8. Christen-Zaech S, Chamlin SL. An 11year-old girl with knuckle plaques. Knuckle pads. Pediatr Ann 2007;36:459-60.

9. Barcaui EO, Carvalho A, Lopes F, et al. High frequency ultrasound with color Doppler in dermatology. An Bras Dermatol 2016;91:262-27.

10. Lopez-Ben R, Dehghanpisheh K, Chatham WW, et al. Ultrasound appearance of knuckle pads. Skeletal Radiol 2006;35:823-7.

11. Chiriac A, Brzezinski P, Foia L, et al. Knuckle Pads - Practical Diagnostic Issues. J Interdiscip Med 2017;2:171174.

12. Esteban-Garrido E, Hernàndez-Nùñez A, Echevarría García B, et al. Ultrasound appareance of a sucking knuckle pad. An update. Eur J Pediatr Dermatol 2019;20-2. 